Myeloid-derived suppressor cells (MDSCs) are a heterogeneous population of early myeloid progenitors and precursors at different stages of differentiation into granulocytes, macrophages, and dendritic cells. Blockade of their differentiation into mature myeloid cells in cancer results in an expansion of this population. Highgrade gliomas are the most common malignant tumours of the central nervous system (CNS), with a poor prognosis despite intensive radiation and chemotherapy. Histopathological and flow cytometry analyses of human and rodent experimental gliomas revealed the extensive heterogeneity of immune cells infiltrating gliomas and their microenvironment. Immune cell infiltrates consist of: resident (microglia) and peripheral macrophages, granulocytes, myeloid-derived suppressor cells, and $\mathrm{T}$ lymphocytes. Intratumoural density of glioma-associated MDSCs correlates positively with the histological grade of gliomas and patient's survival. MDSCs have the ability to attract $T$ regulatory lymphocytes to the tumour, but block the activation of tumour-reactive $\mathrm{CD}^{+}{ }^{+} \mathrm{T}$ helper cells and cytotoxic $C D 8^{+} T$ cells. Immunomodulatory mechanisms employed by malignant gliomas pose an appalling challenge to brain tumour immunotherapy. In this mini-review we describe phenotypic and functional characteristics of MDSCs in humans and rodents, and their occurrence and potential roles in glioma progression. While understanding the complexity of immune cell interactions in the glioma microenvironment is far from being accomplished, there is significant progress that may lead to the development of immunotherapy for gliomas.

Key words: tumour microenvironment, myeloid-derived suppressor cells, antitumor responses, immunosuppression.

Contemp Oncol (Pozn) 2016; 20 (5): 345-351 DOI: $10.5114 /$ wo.2016.64592

\section{Myeloid-derived suppressor cells in gliomas}

\author{
Anna Gieryng, Bozena Kaminska
}

Neurobiology Center, Laboratory of Molecular Neurobiology, Nencki Institute of Experimental Biology, PAS, Warsaw, Poland

\section{Phenotypical and molecular characteristics of myeloid-derived suppressor cells}

Myeloid-derived suppressor cells (MDSCs) are a heterogeneous population of early myeloid progenitors and precursors at different stages of differentiation into granulocytes, macrophages, and dendritic cells. In healthy individuals, immature myeloid cells generated in bone marrow quickly differentiate into mature granulocytes, macrophages, or dendritic cells. Under pathological conditions such as cancer, infectious diseases, sepsis, trauma, bone marrow transplantation, or autoimmune disorders, a blockade in their differentiation into mature myeloid cells results in an expansion of this population [1]. Human MDSCs are identified as HLA-DR-CD11b+CD14-CD33+ cells that express the common myeloid marker CD33, and lack the expression of markers of mature myeloid and lymphoid cells and the MHC-classII molecule HLA-DR [2, 3] (Fig. 1). Moreover, this heterogeneous population is composed of cells defined as CD14+ monocytic MDSC $\left(\mathrm{CD} 11 \mathrm{~b}^{+} \mathrm{CD} 33^{+} \mathrm{C} 14^{+}\right)$ and $\mathrm{CD} 15^{+}$granulocytic MDSC (CD11b+CD33+CD15+CD14-) [4]. Human MDSCs commonly express Siglec-3/CD33 and lack lineage markers and HLA-DR, but heterogeneous expression of CD14 and CD15 suggests the presence of multiple subsets. Several other surface molecules have been used to identify additional subsets of MDSCs in cancer, including CD80, also known as B7.1 [5], CD115 (the macrophage colony-stimulating factor receptor), and CD124 (the IL-4 receptor $\alpha$-chain) $[6,7]$. Although these proteins are expressed by MDSCs, they do not define a specific MDSC population with distinct suppressive functions. Human MDSCs do not express a marker homologous to mouse Gr-1.

In humans, identification of G- and M-MDSC subsets is difficult, they have been described by the combination of several myeloid markers that, in some instances, overlap partially or completely. Additionally, a more immature subset of human MDSCs characterised by the absence of staining for the lineage cocktail (CD3-, CD14- $\left.{ }^{-}, \mathrm{CD}_{15}^{-}, \mathrm{CD}^{-} 9^{-}, \mathrm{CD} 56^{-}\right), \mathrm{HLA}^{-} \mathrm{DR}^{-}$, and expression of the common myeloid markers CD33 and CD11b has been identified as an "early stage" e-MDSC, defined. Using a multi-colour staining protocol and taking into account all the reported myeloid subsets endowed with a suppressive activity, a recent study discriminated six human MDSC phenotypes: MDSC1 (CD14+IL-4R $\left.\alpha^{+}\right)$, MDSC2 (CD15+IL-4R $\left.\alpha^{+}\right)$, MDSC3 (Lineage-HLA-DRCD33+), MDSC4 (CD14+HLA-DR $\left.{ }^{\text {low/- }}\right)$, MDSC5 (CD11b+CD14-CD15+), and MDSC6 (CD15+FSClowSSChigh) (Table 1) [8-10]. The detection of different human MDSC phenotypes highlighted the lack of standardisation in this area and led to the establishment of a human MDSC proficiency panel according to the guidance of the Association of Cancer Immunotherapy (CIMT) Immunoguiding Program in order to promote the standardisation of MDSC immunophenotyping across different groups. The currently accepted phenotypic definitions of human MDSCs are CD11b+CD14+CD33+HLA-DR ${ }^{-1 \text { low }}$ Co-receptor-/low for macrophage M-MDSCs, and CD11b+CD15+CD33+ Lin-HLA-DR-/low for polymononuclear PMN-MDSCS, present in the mononuclear fraction [11]. According to the 


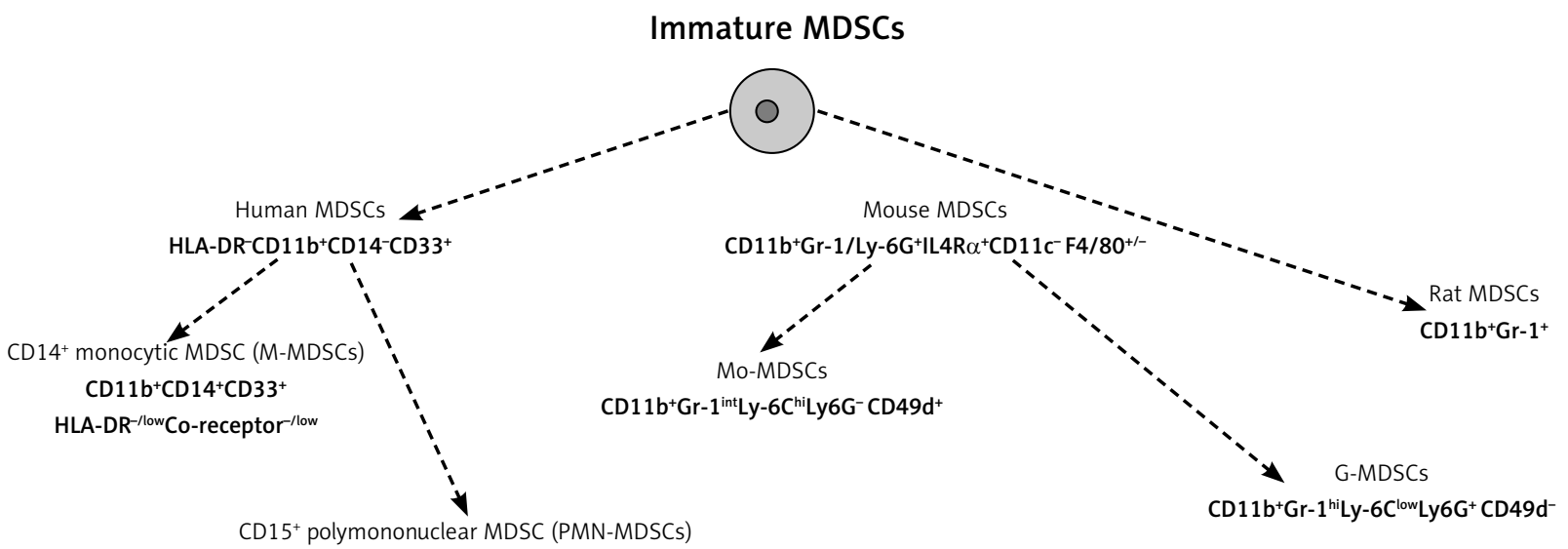

$\mathrm{CD} 11 \mathrm{~b}^{+} \mathrm{CD} 15^{+} \mathrm{CD} 33^{+}$

Lin-HLA-DR-/low Co-receptor/low

Fig. 1. The currently accepted phenotypic definitions of MDSCs

Table 1. Phenotyping of human MDSC subsets with the use of multi-colour staining protocol

\begin{tabular}{lc} 
Human MDSCs & Phenotype (multi-colour staining protocol) \\
MDSC1 & CD14+IL-4R $\alpha^{+}$ \\
MDSC2 & CD15+IL-4R $\alpha^{+}$ \\
MDSC3 & Lineage-HLA-DR-CD33+ \\
MDSC4 & CD14+HLA-DR ${ }^{\text {low/- }}$ \\
MDSC5 & Cd11b ${ }^{+}$CD14-CD15+ \\
MDSC6 & CD15-FSClowSSChigh \\
\hline
\end{tabular}

recommendations the current nomenclature of polymononuclear PMN-MDSCs, present in the mononuclear fraction, replaced former granulocytic G-MDSCs. Growing evidence shows that the phenotype and mechanisms of action of MDSCs are tumour-dependent; therefore, it is important to determine the presence of all MDSC subsets in each cancer patient [12].

In mice, MDSCs are defined as CD11 b+Gr-1/Ly-6G+ IL4R $\alpha^{+} \mathrm{CD} 11 \mathrm{C}^{-} \mathrm{F} 4 / 80^{+/-}$cells [13], but the relative expression levels of Ly- $6 \mathrm{G}$ and Ly-6C also identified two specific subsets: granulocytic (G-MDSCs) CD11b+Gr- ${ }^{\text {hi }}$ Ly-6Clow Ly-6G+CD49dand monocytic (Mo-MDSCs) CD11b+Gr- $1^{\text {int }}$ Ly-6Chi Ly-6G $^{-}$ CD49d $d^{+}$MDSCs [14-16]. Gr-1 antigen is a cell surface protein that belongs to the Ly- 6 family of proteins. In rats MDSCS could be identified as CD11b+Gr-1+ cells (Fig. 2).

MDSCs are of great interest because they have the capacity to suppress the cytotoxic activities of natural killer (NK) and NKT cells, and the adaptive immune response mediated by $\mathrm{CD}^{+}$and $\mathrm{CD} 8^{+} \mathrm{T}$ cells, and they induce apoptosis of T-cell subsets [17]. Multiple mechanisms responsible for MDSC-mediated T-cell suppression have been described. The two MDSC subsets inhibit immune responses through different mechanisms: PNM-MDSCs suppress antigen (Ag)-specific CD8 ${ }^{+} \mathrm{T}$ cells mainly by producing reactive oxygen species (ROS), while M-MDSCs function primarily by expressing nitric oxide synthase 2 (NOS2) and arginase 1 (ARG1), and by generating reactive nitrogen species [18]. ARG1 and NOS2 metabolise L-arginine and block translation of the T cell CD3 zeta chain, inhibit T-cell proliferation, and promote T-cell apoptosis. Moreover, MDSCs secrete immunosuppressive cytokines and induce regulatory T-cell progression. The proportions of PMN-MDSCs and M-MDSCS are variable in different tumour models. While the majority of MDSCs in peripheral lymphoid organs are PMN-MDSCS, the ratio between PMN-MDSCs and M-MDSCS is much lower at tumour sites. It has been proposed that chemokines produced by tumour cells could induce a preferential migration of M-MDSCs to the tumour site. Alternatively, special features of the tumour microenvironment such as hypoxia, low $\mathrm{pH}$, and metabolic products might not support PMN-MDSC survival [18].

Normal bone marrow contains $20-30 \%$ of cells with this phenotype, but these cells constitute only a small proportion (2-4\%) of spleen cells and are absent from the lymph nodes in mice. In healthy individuals, immature myeloid cells with the described above phenotype comprise $\sim 0.5 \%$ of peripheral blood mononuclear cells [3]. Granulocyte-macrophage colony stimulating factor (GM-CSF), granulocyte colony stimulating factor (G-CSF), and macrophage colony stimulating factor (M-CSF) are haematopoietic growth factors responsible for the recruitment, proliferation, and maturation of myeloid cells [19]. One of the main concepts of MSDC generation, the emergency myelopoiesis states that an expansion signal 1 , mediated mainly by STAT3 (induced by, e.g., GM-CSF, G-CSF, and IL-6), mobilises the immature myeloid cells from the bone marrow. An activation signal 2 , mediated mainly by the transcription factor NFKB (induced by pro-inflammatory stimuli, e.g. TLR signalling and cytokines), follows [20]. The concept of block in differentiation states that immature myeloid cells are arrested in their immature phase by inflammatory mediators such as S100A8, S100A9, VEGF, IL-10, and COX-2/ prostaglandin PGE2 [21, 22]. The classical definition of MDSCs as immature myeloid cells blocked from terminal differentiation has been challenged by recent studies suggesting that M-MDSCs and PMN-MDSCs may represent monocytes and granulocytes that have acquired immunosuppressive properties [10]. Both emergency myelopoiesis 

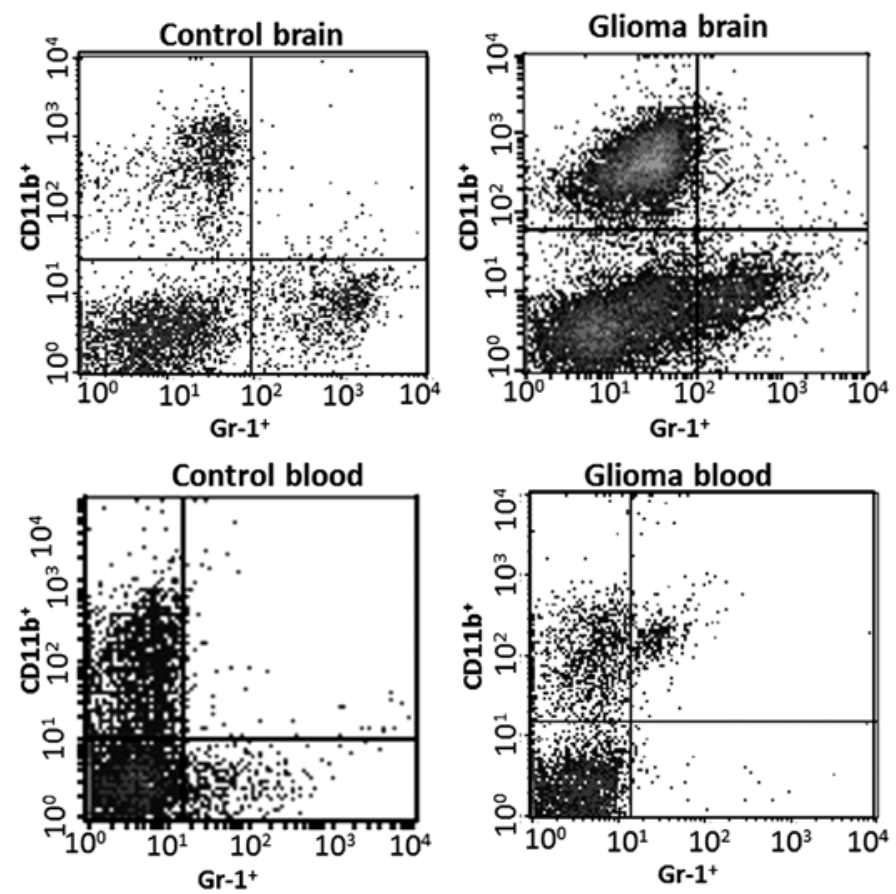

Fig. 2. An example of flow cytometry studies of double positive cells $-\mathrm{CD} 11 \mathrm{~b}+\mathrm{Gr}-1^{+}$from experimental $\mathrm{C} 6$ gliomas

and block in differentiation are linked to an abnormal and persistent activation of STAT3. It is believed that activation of STAT3 in myeloid progenitor cells leads to the induction of S100A8 and S100A9 expression, which subsequently acts in an autocrine manner to arrest the cells in their immature phase [23].

All MDSCs originate from common myeloid progenitors, and their development is probably governed by the same growth factors that control normal myelopoiesis, e.g. GMCSF, G-CSF, and M-CSF. MDSC percentages increase under pathological conditions, as a result of signals released by tumour cells or chronic inflammation [24]. Emerging evidence suggests that M-MDSCs are generated by reprogramming of monocytes into M-MDSCs, whereas PMN-MDSCs might be generated from neutrophils through the activation of immature or mature granulocytes and represent different stages of activation [10].

The frequency of MDSCs positively correlates with clinical stage and tumour burden in patients with breast [25], gastric [26], and colorectal cancers [27]. Increased levels of CD14+HLA-DR-/low MDSCs were correlated with extrathoracic metastasis and poor response to chemotherapy in non-small cell lung cancer patients [28].

The analysis of ten different experimental tumour models revealed that the expansion of the PMN-MDSC population was much greater than that of the monocytic subset, and the two subpopulations used different mechanisms to suppress T-cell function. The ability to differentiate into mature DCs and macrophages in vitro has been shown to be restricted to monocytic M-MDSCs [14]. Several studies have shown that bone marrow precursor cells treated with G-CSF or GM-CSF acquire a surface phenotype similar to MDSCs found in blood of cancer patients [29-31].

\section{Accumulation and functions of myeloid cells in gliomas}

Gliomas are tumours of the central nervous system (CNS), originating from transformed neural stem or progenitor glial cells [32]. The World Health Organisation (WHO), based on histopathological characteristics, divided gliomas into groups: low-grade gliomas (grades I and II) are benign, well-differentiated, slow-growing tumours; whereas high-grade gliomas (grades III and IV) are poorly differentiated or anaplastic, rapidly proliferating, and strongly infiltrate brain parenchyma. The most frequent and malignant primary brain tumour is glioblastoma (GBM, grade IV). Histological classification is currently assisted by molecular genetic features that provide diagnostic, prognostic, and predictive values [33, 34], facilitating patient stratification, prognosis, and treatment response [35]. GBM is one of the most aggressive and difficult to treat human malignancies, due to the frequent dysfunctions of tumour suppressors or oncogenes and highly diffusive growth, which prevents tumour resection and contributes to rapid tumour recurrence [36]. GBMs show alterations in EGFR, PDGFRA, PTEN, TP53, NF1, and CDKN2A/B, TERT promoter mutations, and rarely IDH1/2 mutations (5\%, mainly secondary GBMs that develop from lower grade tumours) $[37,38]$. The golden standard of GBM treatment is surgery combined with chemo- and radiotherapy; however, it remains only palliative and the median survival time of adult patients with GBM is 14-15 months after diagnosis [39].

Glioma cells secrete numerous chemokines, cytokines, and growth factors that promote infiltration of various cells, including a vast majority of immune cells such as microglia, peripheral macrophages, MDSCs, leukocytes, mostly CD4+ $T$ cells, and Treg [40-43]. These non-neoplas- 
tic cells create a specific niche within a tumour microenvironment, which plays an important role in glioma growth, metastasis, and response to treatment. Locally produced cytokines and chemokines and their crosstalk with components of the extracellular matrix re-educate infiltrating immune cells to acquire distinct functional properties, directing the immune system towards immunosuppressive responses. Like many other non-CNS malignant cancers, GBMs developed multiple strategies to inhibit host antitumour responses [44]. Several recent transcriptomic studies of GBM tissues have identified "immune and myeloid/ macrophage" gene expression signatures associated with GBM pathology, overall survival (OS), or response to treatment [45-47]. These data strongly support a link between a type of the immune response, accumulation of specific immune cell subsets, and glioma progression.

Clinical studies showed extensive infiltration of gliomas with myeloid cells; the majority of them are microglia and peripheral macrophages, collectively termed glioma-associated microglia/macrophages (GAMs). Immunohistochemical studies using various markers demonstrated the higher abundance of GAMs in high-grade gliomas than in low-grade gliomas [48-51]. Application of flow cytometry allowed for more detailed analyses of myeloid subpopulations. A flow cytometry phenotype for ramified microglia isolated from adult CNS was defined as CD45 low CD $11 \mathrm{~b} / \mathrm{C}^{+}$ [52-54]. Intratumoural microglia/macrophage density increases during glioma progression and correlates with the grade of malignancy [55-57]. Flow cytometry-based analyses of myeloid cells in experimental gliomas showed accumulation of polarised GAMs within the tumour microenvironment. Using flow cytometry Badie and Schartner determined the proportion of microglia $\left(C D 11 b / c^{\text {high }}\right.$ CD45 low $)$, macrophages (CD11b// $\left.{ }^{\text {high }} C D 45^{\text {high }}\right)$, and lymphocytes (CD11b/c-CD45 high) in rat C6, 9L, and RG-2 glioma models, and demonstrated that microglia infiltration is dependent on the glioma cell line but does not correlate with the tumour size [54]. Microglia accounted for 13-34\% of the tumour mass within the tumour periphery. Macrophages represented a smaller proportion of the infiltrating cells, accounting for $5-12 \%$ of cells, and this proportion was constant in different models [54]. In the transgenic RCAS-PDGFb tumour model in which $\mathrm{RFP}^{-} / \mathrm{GFP}^{+}$microglia and $\mathrm{RFP}^{+} /$GFPlow macrophages/monocytes were isolated from tumours, flow cytometry studies demonstrated $14 \%$ microglia and $8.5 \%$ macrophages/monocytes in the RCAS-PDGFb gliomas, and a similar proportion of GAMs (16\% microglia and 6.5\% macrophages/monocytes) was observed in GL261 tumours [58].

Immunohistochemical evaluation of functional markers and gene expression profiling of human GBM infiltrating $\mathrm{CD}_{11 \mathrm{~b}^{+}}$cells indicate immunosuppressive activation of GAMs and point to a lack of the classical "immune response" activation in GBMs [51, 58-60]. The studies using pharmacological or genetic ablation of CD11b+ cells in experimental glioma models demonstrated that tumours do not grow and invade the brain parenchyma if GAMs are ablated or functionally paralysed [59, 61-66]. In those studies the percentages of MDSCs and their functionality were not evaluated.

\section{Myeloid-derived suppressor cells in gliomas}

The numbers of MSDCs are frequently increased in blood, spleen, and tumour mass, and they correlate with cancer stage, metastasis, and chemotherapy response [18]. Information regarding their presence and roles in gliomas is scarce $[4,67]$. Gielen et al. [4] reported increased percentages of both M-MDSCs and PMN-MDSCs in the blood of GBM patients when compared with healthy donors. The myeloid activation markers B7-1/CD80 and PDL1 were not detected, and CD124, CD86, and CD40 were detected at similar levels on MDSCs in glioma patients and healthy donors. The MDSC population in tumour cell suspensions consisted almost exclusively of CD15+ PMNMDSC cells. Immunohistochemistry confirmed infiltration of glioma tissues with CD15+/HLAll- cells [4]. Raychaudhuri et al. reported that patients with GBM have elevated levels of MDSCs compared with age-matched healthy donors and other cancer patients. The majority of the MDSCs in GBM patients were CD15+ CD14- PMN-MDSCs (82\%), followed by lineage-negative e-MDSCs (15\%) and M-MDSCs (3\%) [67]. Another study of 52 GBM patients revealed a significantly higher frequency of $\mathrm{CD} 14^{\text {high }} \mathrm{CD} 15^{+} \mathrm{M}-\mathrm{MD}$ SCS and CD14 low CD15+ PMN-MDSCs in blood of GBM patients when compared with healthy controls. Correlation between the number of PMN-MDSCS and CD4+ effector memory T-cells $\left(C D 4^{+} T_{\text {em }}\right)$ within the tumours was detected. Tumour-derived $C^{2} 4^{+} T_{\text {em }}$ expressed high levels of PD-1 and was functionally exhausted. The expression of PDL1 was also significantly upregulated on tumour-derived MDSCs [68]. The presented findings provide evidence for the accumulation of different MDSC subsets in GBM patients and indicate that PMN-MDSCs in peripheral blood and at the tumour site may participate in GBM-induced T-cell suppression. Accumulation of MDSCs in peripheral blood in GBM patients may induce T-cell immunosuppression, and increased plasma levels of Arginase 1 and G-CSF may contribute to MDSC suppressor function and its expansion [4].

These results are corroborated by our studies, which show a large number of $\mathrm{Arg}^{+}$but not Iba1 ${ }^{+}$cells (GAMs) infiltrating experimental C6 gliomas in rats [66]. Flow cytometry studies of double-positive cells $-\mathrm{CD} 11 \mathrm{~b}^{+} \mathrm{Gr}-\mathrm{1}^{+}$ from experimental C6 gliomas (Fig. 1B) show a negligible number of such cells in normal brain and an increase of up to $3-4 \%$ in the tumour-bearing hemispheres. In addition, an increased percentage of CD11 b+Gr-1+ cells was detected in the peripheral blood (5.5-6.7\%) of animals implanted with glioma cells (unpublished). It confirms the increase of MDSCs in experimental rat gliomas.

Recent studies indicate that GAMs (in particular CD163+ infiltrating macrophages) within the glioma microenvironment produce $\mathrm{Ccl} 2$, a chemokine recruiting both $\mathrm{CCr}^{+}{ }^{+} \mathrm{Ly}-6 \mathrm{C}^{+} \mathrm{Mo}-\mathrm{MDSCS}$ and $\mathrm{Cr} 4^{+}$Treg. In murine gliomas, tumour-derived $\mathrm{Cc} 20$ and osteoprotegerin induced $\mathrm{Cl} 2$ production by GAMs. Gliomas developing in $\mathrm{Ccl} 2$-deficient mice displayed reduced Tregs and monocytic MDSCs infiltration [69]. Macrophage migration inhibitory factor (MIF), which was produced at high levels by glioma stem-like cells (CSCS), increased the expression of the arginase- 1 in 
MDSCs in a receptor Cxcr2-dependent manner. Reduction of MIF conferred a survival advantage to tumour-bearing animals and increased the cytotoxic $T$ cell response towards the tumour [70]. M-MDSCs of GBM patients have increased levels of intracellular S100A8/9 and serum levels compared with M-MDSCs in healthy controls. Glioma patients also have increased S100A8/9 serum levels, which correlates with increased Arginase activity in serum [71].

\section{Antitumour immune responses in glioma microenvironment}

Accumulation of GAMs and MDSCs, and their functional polarisation into pro-invasive and immunosuppressive cells, have a profound effect on antitumour responses in gliomas. The glioma microenvironment is infiltrated with leukocytes, mostly CD4+ $T$ helper (Th), CD8 ${ }^{+} T$ cytotoxic (Tc), and $\mathrm{CD}^{+}{ }^{+} \mathrm{CD} 25^{+} \mathrm{FoxP}^{+}$Treg $[40-42,55,72,73]$. The presence of $\mathrm{CD}^{+}{ }^{+} \mathrm{CD} 25^{+} \mathrm{FoxP}^{+}$Treg was correlated with tumour resistance to radiation and chemotherapy, poor prognosis, and higher grade $[43,44]$. Treg cells are known as suppressors of the adaptive immune response inhibiting the proliferation of effector T cells. Treg suppressive mechanism depends on the cell-cell interaction and is implicated in a host tolerance in tumour growth [55]. The tumour-infiltrating CD8+ $T$ cells were phenotypically $C D 8^{+} C D 25^{-}$, suggesting that these cells were not activated. $\mathrm{CD}^{+} \mathrm{T}$ cells were more numerous than $\mathrm{CD}^{+} \mathrm{T}$ cells within glioma tissues, and the percentages of $\mathrm{CD}^{+}$and $\mathrm{CD} 8^{+}$cells increased with tumour grade $[74,75]$.

Anti-tumour immune responses mediated by $T$ cells are suppressed by TGF- $\beta$ and IL-10 secreted by glioma cells [44]. Moreover, glioma cells lack B7.1/2 (CD80/86), costimulatory and immune regulatory molecules, but constitutively express $\mathrm{B} 7-\mathrm{H} 1$ mRNA and protein. Glioma-related $\mathrm{B} 7-\mathrm{H} 1$ was identified as a strong inhibitor of $\mathrm{CD}^{+}{ }^{+}$as well as $\mathrm{CD} 8^{+} \mathrm{T}$-cell activation [75]. GBM infiltrating T-cells had decreased INF- $\gamma$ production and increased $P D-1$ gene expression [68]. As well as directly blocking effective activation of naive T cells, glioma cells promote accumulation of Treg and MDSCs that mediate inhibition of T cells and NK cells.

Immunotherapeutic approaches for GBM, such as immune checkpoint blockade and anti-tumour vaccines, are actively tested in preclinical models and the first combinations of immunotherapies with standard therapies are being translated to clinical trials. Phase I clinical trial studying the effects of anti-PD-1 and anti-CTLA-4 combination therapy for recurrent GBM (NCT02017717), and a number of studies of DC vaccines in recurrent and newly diagnosed GBM are in progress (NCT02010606, NCT02149225, NCT02049489, NCT01808820, NCT02078648), and an entire medical community awaits results. Further understanding the biology of MDSCs and conditions for their polarisation in the tumour microenvironment and peripheral blood may be crucial for the development of new therapeutic strategies.

\section{Acknowledgements}

Studies supported by the project DIMUNO: "Development of new cancer therapies based on selective antitumor immunomodulators" - co-financed by the National Centre for Research and Development in the framework of STRATEGMED-3 Prevention practices and treatment of civilisation diseases.

The authors declare no conflict of interest.

\section{References}

1. Kusmartsev S, Gabrilovich DI. Inhibition of myeloid cell differentiation in cancer: The role of reactive oxygen species. J Leukoc Biol 2003; 74: 186-96.

2. Almand B, Clark JI, Nikitina E, van Beynen J, English NR, Knight SC, Carbone DP, Gabrilovich DI. Increased production of immature myeloid cells in cancer patients: a mechanism of immunosuppression in cancer. J Immunol 2001; 166: 678-89.

3. Nagaraj S, Gabrilovich DI. Myeloid-derived suppressor cells in human cancer. Cancer J. 2010; 16: 348-53.

4. Gielen PR, Schulte BM, Kers-Rebel ED, Verrijp K, Petersen-Baltussen HM, ter Laan M, Wesseling P, Adema GJ. Increase in both CD14-positive and CD15-positive myeloid-derived suppressor cell subpopulations in the blood of patients with glioma but predominance of CD15-positive myeloid-derived suppressor cells in glioma tissue. J Neuropathol Exp Neurol 2015; 74: 390-400.

5. Yang R, Cai Z, Zhang Y, Yutzy WH 4th, Roby KF, Roden RB. CD80 in immune suppression by mouse ovarian carcinoma-associated $\mathrm{Gr}$ 1+CD11b+ myeloid cells. Cancer Res 2006; 66: 6807-15.

6. Huang B, Pan PY, Li Q, Sato AI, Levy DE, Bromberg J, Divino CM, Chen SH. Gr-1+CD115+ immature myeloid suppressor cells mediate the development of tumor-induced T regulatory cells and T-cell anergy in tumor-bearing host. Cancer Res 2006; 66: 1123-31.

7. Gallina G, Dolcetti L, Serafini P, et al. Tumors induce a subset of inflammatory monocytes with immunosuppressive activity on CD8+ T cells. J Clin Invest 2006; 116: 2777-90.

8. Dumitru CA, Moses K, Trellakis S, Lang S, Brandau S. Neutrophils and granulocytic myeloid-derived suppressor cells: immunophenotyping, cell biology and clinical relevance in human oncology. Cancer Immunol Immunother 2012; 61: 1155-67.

9. Talmadge JE, Gabrilovich DI. History of myeloid-derived suppressor cells. Nat Rev Cancer 2013; 13: 739-52.

10. Millrud CR, Bergenfelz C, Leandersson K. On the origin of myeloid-derived suppressor cells. Oncotarget. 2016 Sep 27. doi: 10.18632/oncotarget.12278. [Epub ahead of print]

11. Bronte V, Brandau S, Chen SH, Colombo MP, Frey AB, Greten TF, Mandruzzato S, Murray PJ, Ochoa A, Ostrand-Rosenberg S, Rodriguez PC, Sica A, Umansky V, Vonderheide RH, Gabrilovich DI. Recommendations for myeloid-derived suppressor cell nomenclature and characterization standards. Nat Commun 2016; 7: 12150.

12. Solito S, Marigo I, Pinton L, Damuzzo V, Mandruzzato S, Bronte V. Myeloid-derived suppressor cell heterogeneity in human cancers. Ann N Y Acad Sci 2014; 1319: 47-65.

13. Gabrilovich DI, Ostrand-Rosenberg S, Bronte V. Coordinated regulation of myeloid cells by tumours. Nat Rev Immunol 2012; 12: 253-68.

14. Youn JI, Nagaraj S, Collazo M, Gabrilovich DI. Subsets of myeloid-derived suppressor cells in tumor-bearing mice. J Immunol 2008; 181: 5791-802.

15. Dolcetti L, Peranzoni E, Ugel S, et al. Hierarchy of immunosuppressive strength among myeloid-derived suppressor cell subsets is determined by GM-CSF. Eur I Immunol 2010; 40: 22-35.

16. Haile LA, Gamrekelashvili J, Manns MP, Korangy F, Greten TF. CD49d is a new marker for distinct myeloid-derived suppressor cell subpopulations in mice. J Immunol 2010; 185: 203-10.

17. Marvel D, Gabrilovich DI. Myeloid-derived suppressor cells in the tumor microenvironment: expect the unexpected. J Clin Investig 2015; 125: 3356-64.

18. Youn JI, Gabrilovich DI. The biology of myeloid-derived suppressor cells: the blessing and the curse of morphological and functional heterogeneity. Eur J Immunol 2010; 40: 2969-75. 
19. Hamilton JA, Achuthan A. Colony stimulating factors and myeloid cell biology in health and disease. Trends in Immunology 2013; 34: 81-9.

20. Condamine T, Mastio J, Gabrilovich DI. Transcriptional regulation of myeloid-derived suppressor cells. J Leukoc Biol 2015; 98: 913 22.

21. Obermajer N, Muthuswamy R, Lesnock J, Edwards RP, Kalinski P. Positive feedback between PGE2 and COX2 redirects the differentiation of human dendritic cells toward stable myeloid-derived suppressor cells. Blood 2011; 118: 5498-505.

22. Mao Y, Poschke I, Wennerberg E, et al. Melanoma-educated CD14+ cells acquire a myeloid-derived suppressor cell phenotype through COX-2-dependent mechanisms. Cancer Res 2013; 73 3877-87.

23. Cheng P, Corzo CA, Luetteke N, et al. Inhibition of dendritic cell differentiation and accumulation of myeloid-derived suppressor cells in cancer is regulated by S100A9 protein. J Exp Med 2008; 205: 2235-49.

24. Kumar V, Patel S, Tcyganov E, Gabrilovich DI. The Nature of myeloid-derived suppressor cells in the tumor microenvironment. Trends Immunol 2016; 37: 208-20.

25. Bergenfelz C, Larsson AM, von Stedingk K, et al. Systemic monocytic-MDSCs are generated from monocytes and correlate with disease progression in breast cancer patients. PLoS One 2015; 10: e0127028.

26. Wang L, Chang EW, Wong SC, Ong SM, Chong DQ, Ling KL. Increased myeloid-derived suppressor cells in gastric cancer correlate with cancer stage and plasma S100A8/A9 proinflammatory proteins. J Immunol 2013; 190: 794-804.

27. Sun HL, Zhou X, Xue YF, Wang K, Shen YF, Mao JJ, Guo HF, Miao ZN. Increased frequency and clinical significance of myeloid-derived suppressor cells in human colorectal carcinoma. World J Gastroenterol 2012; 18: 3303-9.

28. Huang A, Zhang B, Wang B, Zhang F, Fan KX, Guo YJ. Increased CD14(+)HLA-DR (-/low) myeloid-derived suppressor cells correlate with extrathoracic metastasis and poor response to chemotherapy in non-small cell lung cancer patients. Cancer Immu nol Immunother 2013; 62: 1439-51.

29. Pak AS, Wright MA, Matthews JP, Collins SL, Petruzzelli GJ, Young MR. Mechanisms of immune suppression in patients with head and neck cancer: presence of CD34(+) cells which suppress im mune functions within cancers that secrete granulocyte-macro phage colony-stimulating factor. Clin Cancer Res 1995; 1: 95-103.

30. Abrams SI, Waight JD. Identification of a G-CSF-Granulocytic MDSC axis that promotes tumor progression. Oncoimmunology 2012; 1: 550-551.

31. Annels NE, Shaw VE, Gabitass RF, et al. The effects of gemcitabine and capecitabine combination chemotherapy and of low-dose adjuvant GM-CSF on the levels of myeloid-derived suppressor cells in patients with advanced pancreatic cancer. Cancer Immunol Immunother 2014; 63: 175-83.

32. Ilkanizadeh S, Lau J, Huang M, et al. Glial progenitors as targets for transformation in glioma. Adv Cancer Res 2014; 121: 1-65.

33. Weller M, Stupp R, Hegi ME, van den Bent M, Tonn JC, Sanson M, Wick W, Reifenberger G. Personalized care in neuro-oncology coming of age: Why we need MGMT and $1 p / 19 q$ testing for malignant glioma patients in clinical practice. Neuro Oncol 2012; 14 Suppl 4: iv100-8.

34. Weller M, Pfister SM, Wick W, Hegi ME, Reifenberger G, Stupp R. Molecular neuro-oncology in clinical practice: A new horizon. Lancet Oncol 2013; 14: e370-9.

35. Ceccarelli M, Barthel FP, Malta TM, et al. Molecular profiling re veals biologically discrete subsets and pathways of progression in diffuse glioma. Cell 2016; 164: 550-63.

36. Sarin $H$. Recent progress towards development of effective sys temic chemotherapy for the treatment of malignant brain tu mors. J Transl Med 2009; 7: 77.

37. Tabatabai G, Stupp R, van den Bent MJ, Hegi ME, Tonn JC, Wick W, Weller M. Molecular diagnostics of gliomas: The clinical perspective. Acta Neuropathologica 2010; 120: 585-92.

38. Vigneswaran K, Neill S, Hadjipanayis CG. Beyond the World Health Organization grading of infiltrating gliomas: advances in the molecular genetics of glioma classification. Ann Transl Med 2015; 3: 95.

39. Van Meir EG, Hadjipanayis CG, Norden AD, Shu HK, Wen PY, OIson JJ. Exciting New Advances in Neuro-Oncology: The Avenue to a Cure for Malignant Glioma. CA. Cancer J Clin 2010; 60: 166-93.

40. Fecci PE, Mitchell DA, Whitesides JF. Increased regulatory T-cell fraction amidst a diminished CD4 compartment explains cellular immune defects in patients with malignant glioma. Cancer Res 2006; 66: 3294-302.

41. Lohr J, Ratliff T, Huppertz A. Effector T-cell infiltration positively impacts survival of glioblastoma patients and is impaired by tumor-derived TGF-? Clin Cancer Res 2011; 17: 4296-308.

42. Alexiou GA, Vartholomatos G, Karamoutsios A, Batistatou A, Kyritsis AP, Voulgaris S. Circulating progenitor cells: A comparison of patients with glioblastoma or meningioma. Acta Neurol Belg 2013; 113: 7-11.

43. Wainwright DA, Dey M, Chang, A, Lesniak MS. Targeting tregs in malignant brain cancer: Overcoming IDO. Front Immunol 2013; 15: 116

44. Perng P, Lim M. Immunosuppressive mechanisms of malignant gliomas: parallels at non-CNS Sites. Front Oncol 2015; 5: 153.

45. Godard S, Getz G, Delorenzi M, et al. Classification of human astrocytic gliomas on the basis of gene expression: a correlated group of genes with angiogenic activity emerges as a strong predictor of subtypes. Cancer Res 2003; 63: 6613-25.

46. Shirahata M, Iwao-Koizumi K, Saito S, Ueno N, Oda M, Hashimoto N, Takahashi JA, Kato K. Gene expression-based molecular diagnostic system for malignant gliomas is superior to histological diagnosis. Clin Cancer Res 2007; 13: 7341-56.

47. Vauléon E, Tony A, Hamlat A, et al. Immune genes are associated with human glioblastoma pathology and patient survival. BMC Med Genomics 2012; 5: 41.

48. Esiri M, Morris C. Immunocytochemical study of macrophages and microglial cells and extracellular matrix components in hu man CNS disease. 2. Non-neoplastic diseases. J Neurol Sci 1991; 101: $59-7$

49. Wierzba-Bobrowicz, T, Kuchna, I, Matyja E. Reaction of microglial cells in human astrocytomas (preliminary report). Folia Neuropathol 1994; 32: 251-2.

50. Geranmayeh F, Scheithauer BW, Spitzer C, Meyer FB, Svensson-Engwall AC, Graeber MB. Microglia in gemistocytic astrocytomas. Neurosurgery 2007; 60: 159-66.

51. Mieczkowski J, Kocyk M, Nauman P, et al. Down-regulation of IKK $\beta$ expression in glioma-infiltrating microglia/macrophages is associated with defective inflammatory/immune gene responses in glioblastoma. Oncotarget 2015; 6: 33077-90.

52. Sedgwick JD, Schwender S, Imrich H, Dörries R, Butcher GW, te Meulen V. Isolation and direct characterization of resident microglial cells from the normal and inflamed central nervous system. Proc Natl Acad Sci U S A 1991; 88, 7438-42.

53. Sedgwick JD, Schwender S, Gregersen R, Dörries R, ter Meulen V. Resident macrophages (ramified microglia) of the adult brown Norway rat central nervous system are constitutively major histocompatibility complex class II positive. J Exp Med 1993; 177: 1145-52.

54. Badie, B, Schartner, JM. Flow cytometric characterization of tumor-associated macrophages in experimental gliomas. Neurosurgery 2000; 46: 957-62.

55. Hussain SF, Yang D, Suki D, Aldape K, Grimm E, Heimberger AB. The role of human glioma-infiltrating microglia/macrophages in mediating antitumor immune responses. Neuro Oncol 2006; 8: 261-79.

56. Badie B, Schartner J. Role of microglia in glioma biology. Microsc Res Tech 2001; 54: 106-13.

57. Yi L, Xiao H, Xu M, et al. Glioma-initiating cells: A predominant role in microglia/macrophages tropism to glioma. J Neuroimmunol 2011; 232: 75-82.

58. Szulzewsky F, Pelz A, Feng X, et al. Glioma-associated microglia/ macrophages display an expression profile different from M1 and M2 polarization and highly express Gpnmb and Spp1. PLoS One 2015; 10: e0116644. 
59. Gabrusiewicz K, Rodriguez B, Wei J, et al. Glioblastoma-infiltrated innate immune cells resemble $\mathrm{MO}$ macrophage phenotype. $\mathrm{JCl}$ insight 2016; 1: 1-32.

60. Szulzewsky F, Arora S, de Witte L, et al. Human glioblastoma-associated microglia/monocytes express a distinct RNA profile com pared to human control and murine samples. Glia Glia 2016; 64: 1416-36.

61. Sliwa M, Markovic D, Gabrusiewicz K, et al. The invasion promot ing effect of microglia on glioblastoma cells is inhibited by cyclosporin A. Brain 2007; 130 (Pt 2): 476-89.

62. Wesolowska A, Kwiatkowska A, Slomnicki L, et al. Microglia-de rived TGF- $\beta$ as an important regulator of glioblastoma invasion - an inhibition of TGF- $\beta$-dependent effects by shRNA against human TGF- $\beta$ type II receptor. Oncogene 2007; 27: 918-30.

63. Markovic DS, Vinnakota K, Chirasani S, Synowitz M, Raguet $H$, Stock K, Sliwa M, Lehmann S, Kälin R, van Rooijen N, Holmbeck K, Heppner FL, Kiwit J, Matyash V, Lehnardt S, Kaminska B, Glass R, Kettenmann H.: Gliomas induce and exploit microglial MT1-MMP expression for tumor expansion. Proc Natl Acad Sci U S A 2009; 106: $12530-5$

64. Gabrusiewicz K, Hossain MB, Cortes-Santiago N, Fan X, Kaminska B, Marini FC, Fueyo J, Gomez-Manzano C. Macrophage ablation reduces $M 2$-like populations and jeopardizes tumor growth in a MAFIA-based glioma model. Neoplasia 2015; 17: 374-84.

65. Gabrusiewicz K, Ellert-Miklaszewska A, Lipko M, Sielska M, Frankowska M, Kaminska B. Characteristics of the alternative phenotype of microglia/macrophages and its modulation in experimental gliomas. PLoS One 2011; 6: e23902.

66. Ellert-Miklaszewska A, Wisniewski P, Kijewska M, et al. Tumour-processed osteopontin and lactadherin drive the protu morigenic reprogramming of microglia and glioma progression. Oncogene 2016; 4. doi: 10.1038/onc.2016.55.

67. Raychaudhuri B, Rayman P, Ireland J, et al. Myeloid-derived suppressor cell accumulation and function in patients with newly diagnosed glioblastoma. Neuro Oncol 2011; 13: 591-9.

68. Dubinski D, Wölfer J, Hasselblatt M, Schneider-Hohendorf T, Bogdahn U, Stummer W, Wiendl H, Grauer OM. CD4+ T effector memory cell dysfunction is associated with the accumulation of granulocytic myeloid-derived suppressor cells in glioblastoma patients. Neuro-Oncology 2016; 18: 807-18.

69. Chang AL, Miska J, Wainwright DA, et al. CCL2 Produced by the glioma microenvironment is essential for the recruitment of regulatory T cells and myeloid-derived suppressor cells. Cancer Res 2016; 76: 5671-82.

70. Otvos B, Silver DJ, Mulkearns-Hubert EE, et al. Cancer stem cell-secreted macrophage migration inhibitory factor stimulates myeloid derived suppressor cell function and facilitates glioblastoma immune evasion. Stem Cells 2016; 34: 2026-39.

71. Gielen PR, Schulte BM, Kers-Rebel ED, Verrijp K, Bossman SA, Ter Laan M, Wesseling P, Adema GJ.: Elevated levels of polymorpho nuclear myeloid-derived suppressor cells in patients with glioblastoma highly express S100A8/9 and arginase and suppress T cell function. Neuro Oncol 2016; 18: 1253-64.

72. Wainwright DA, Sengupta S, Han Y, Lesniak MS. Thymus-derived rather than tumor-induced regulatory $T$ cells predominate in brain tumors. Neuro Oncol 2011; 13: 1308-23.

73. Heimberger AB, Abou-Ghazal M, Reina-Ortiz C, Yang DS, Sun W, Oiao W, Hiraoka N, Fuller GN. Incidence and prognostic impact of FoxP3+ regulatory T cells in human gliomas. Clin. Cancer Res 2008; 14: 5166-72.

74. Kmiecik J, Poli A, Brons NH, Waha A, Eide GE, Enger P $\varnothing$, Zimmer J, Chekenya M. Elevated CD3+ and CD8+ tumor-infiltrating immune cells correlate with prolonged survival in glioblastoma patients despite integrated immunosuppressive mechanisms in the tumor microenvironment and at the systemic level. J Neuroimmu nol 2013; 264: 71-83.

75. Wintterle S, Schreiner B, Mitsdoerffer M, Schneider D, Chen L, Meyermann R, Weller M, Wiendl H. Expression of the B7-related molecule B7-H1 by glioma cells: a potential mechanism of immune paralysis. Cancer Res 2003; 63: 7462-7.

\section{Address for correspondence}

\section{Anna Gieryng}

Laboratory of Molecular Neurobiology

Neurobiology Center

Nencki Institute of Experimental Biology, PAS

Pasteura 3

02-093 Warsaw, Poland

e-mail: a.gieryng@nencki.gov.pl

Submitted: 27.10 .2016

Accepted: $\quad 25.11 .2016$ 